\title{
EVALUACIÓN DE UNA NUEVA TRAMPA PARA CAPTURAR CONEJOS SILVESTRES (Sylvilagus cunicularius y S. floridanus)
}

\section{JORGE VÁZQUEZ ${ }^{1}$, LUISA RODRÍGUEZ-MARTÍNEZ1, AMANDOBAUTISTA", ROBYN HUDSON² Y MARGARITA MARTÍNEZ-GÓMEZ ${ }^{1,2}$}

\author{
${ }^{1}$ Centro Tlaxcala de Biología de la Conducta, Universidad Autónoma de Tlaxcala. Apartado \\ Postal 262. Tlaxcala. 90070 México. \\ ${ }^{2}$ Departamento de Biología Celular y Fisiología. Instituto de Investigaciones Biomédicas, \\ UNAM. Apartado Postal 70228. México. D.F. 04510 México. \\ correo electrónico:marmagabo@yahoo.com,abopup@gmail.com
}

\begin{abstract}
Resumen: En este artículo se describe el diseño de una trampa de red que se desarrolló para capturar conejos silvestres de las especies Sylvilagus cunicularius y S. floridanus en el Parque Nacional La Malinche, Tlaxcala. El desempeño de la trampa de red fue comparado con el de trampas de caja evaluando la eficiencia de captura y recaptura, el sesgo de capturas respecto al sexo y peso corporal y los tipos de lesiones que causan cada tipo de trampa sobre los conejos durante su captura. Se obtuvo mayor eficiencia de captura y recaptura de $S$. cunicularius con las trampas de red que con las de caja y no se encontraron sesgos de captura respecto al sexo en cada tipo de trampa. Sólo en trampas de red hubo un sesgo de captura hacia los individuos más pesados de $S$. cunicularius. En cambio, hubo un sesgo de capturas respecto al sexo en $S$. floridanus (fueron capturados una mayor proporción de machos), no se lograron observar los patrones encontrados en $S$. cunicularius, posiblemente porque $S$. floridanus fue la especie menos representada en las capturas. En ambas especies de conejos, las lesiones causadas durante su captura fueron más severas cuando se capturaron en trampas de caja que en las de red. Se concluye que la trampa de red es un buen dispositivo para realizar capturas y recapturas de conejos silvestres del género Sylvilagus, preferentemente conejos de mayor peso sobre todo en aquellos casos que se opte por capturar individuos adultos, tanto hembras como machos. Además este tipo de trampa de red reduce daños físicos en los conejos atrapados.
\end{abstract}

Palabras clave: Conejos, Sylvilagus cunicularius, S. floridanus, trampa de red, trampa de caja, eficiencia de captura, Tlaxcala.

Abstract: We describe the design of a net trap developed to capture wild rabbits of the species
Silvilagus cunicularius and S. floridanus in La Malinche National Park, Tlaxcala. The perform-
ance of the net trap was compared with that of box traps, evaluating the efficiency of capture
and recapture, and bias with respect to sex, body mass and the type of injuries associated with
capture using each method. For S. cunicularius greater efficiency of capture and recapture was
obtained with the net traps than with the box traps, no bias was found for sex for either type
of trap, and only a bias was found towards the capture of the heaviest animals for the net traps.
For S. floridanus, although there was a bias in captures with respect to sex (captures comprised 
a greater proportion of males), the pattern of captures was not as clear as for S. cunicularius, possibly due to the smaller number of captures for this species. In both species injuries caused by capture were more severe for the box than for the net traps. In conclusion, the net trap described here is a good method for capturing and recapturing wild rabbits of the genus Sylvilagus, and particularly heavier adult animals of either sex. It also reduces physical damage to the trapped animals.

Key words: Rabbits, Sylvilagus cuniculus, S. floridanus, net traps, box traps, trapping efficiency, Tlaxcala.

\section{INTRODUCCIÓN}

La captura de mamíferos silvestres ha sido una estrategia muy importante en estudios científicos para conocer la biología de varias especies así como para desarrollar programas efectivos de conservación (Luengos-Vidal et al., 2003). Muchos estudios sobre el manejo de vida silvestre, conservación e investigación requieren aún la captura de animales por varias razones como transferencia, marcaje y colocación de collares. Actualmente se señala que los investigadores están obligados a frenar los efectos negativos que ocurren durante la manipulación de los animales en sus estudios (Powell y Proulx, 2003). Por tanto, los investigadores deben usar trampas que sean eficientes pero que reduzcan los daños físicos y el estrés de los animales capturados.

Existe una amplia diversidad de trampas para capturar vivos a animales silvestres y pueden ser muy selectivas. Sin embargo, emplearlas en animales para los cuales no fueron diseñadas puede llevar a una tasa de captura reducida y, lo más grave, a un aumento de las lesiones corporales, el estrés e incluso la muerte de los animales (Powell y Proulx, 2003). Los investigadores Powell y Proulx (2003) revisaron los métodos de captura de mamíferos para investigaciones y proponen trampas para capturar algunas especies. Otra forma de reducir los daños de los animales, sin dejar de poner atención sobre incrementar la eficiencia de captura, es mejorar o diseñar métodos de trampeo específicos para cada especie de interés.

Del elevado número de especies de lagomorfos que se reportan en México (Cervantes, 1993), casi la mitad está bajo algún estatus de conservación y de éstas la mayoría se cataloga en riesgo de extinción (Ceballos, 2005). Pero todavía hay desconocimiento en muchas especies de lagomorfos de algunas características como tamaño de poblaciones, abundancia y áreas de distribución lo cual hace necesario el estudiarlos y por lo que se requieren frecuentes capturas y recapturas de los individuos vivos a lo largo de ciertos períodos (González et al., 2007; Vázquez et al., 2007).

El uso de métodos que resulten en una baja tasa de recapturas o en el deceso de los individuos podría provocar la pérdida de valiosa información. Un método común para la captura de conejos silvestres vivos es utilizar trampas de caja (Vanzolini 
y Papayero, 1990), tanto en estudios científicos (e.g. Chapman y Trethewey, 1972), como cuando son considerados una plaga (Craven, 1994; Thurston y Brittingham, 1997). La caja trampa es un buen diseño para la captura de conejos silvestres vivos pero cuando están constituidas de malla metálica algunas veces pueden causar severas lesiones a los conejos (J. Vázquez, com. pers.).

Como parte de un proyecto más amplio sobre la biología de los conejos silvestres de Tlaxcala (González et al., 2007; Vázquez et al., 2007), se implementó y evaluó un nuevo diseño de trampa para la captura de conejos vivos Sylvilagus cunicularius y $S$. floridanus que de aquí en adelante llamaremos trampa red. Por tanto, en este reporte se describe el diseño de esta trampa de red comparando su eficiencia de captura con la de las trampas de caja.

\section{MÉTODOS}

\section{Sitio de estudio}

El estudio fue realizado en 10 hectáreas a $9.5 \mathrm{~km}$ al oeste del municipio de Ixtenco, en

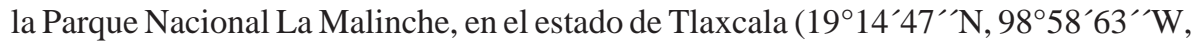
2900-m). Una descripción detallada del sitio de estudio se muestra en Vázquez et al., (2007). Para realizar las capturas de los conejos se eligieron tres sitios de aproximadamente 0.5 hectáreas con una separación de 0.5 km entre cada sitio.

\section{Diseño de la trampa de red}

La trampa de red está constituida de una malla de plástico (con abertura de $1 \mathrm{~mm}^{2}$ ) de $3 \times 3$ metros con una liga de plástico unida a su perímetro, un tubo metálico (calibre 3/4 pulgadas) de $3 \mathrm{~m}$ de longitud con cuatro ligas unidas a uno de sus extremos en el centro de la red (Figura 1), hilos de monofilamento que salen de cada esquina de la red para unirse a un hilo de monofilamento del disparador (Figura 1) y de un sistema de disparo de acción manual (Figura 1).

Al instalar la trampa de red, la malla de plástico se extiende sobre el suelo sosteniéndose cada una de sus esquinas con una estaca de metal clavada al suelo. El tubo se coloca en el centro de la red y se sostiene con tres hilos de monofilamento. Las ligas de plástico de la punta del tubo de metal (Figura 1a) se estiran para ser enganchadas a una de las esquinas de la malla (Figura 1b). De cada esquina de la malla se sujeta un hilo de monofilamento que a su vez se sujeta al sistema de disparo. El disparador se coloca a un lado de la trampa a una distancia de aproximadamente seis metros de la red y se fija al suelo con una estaca de metal (Figura 1c). Cuando se quiere activar la trampa (porque un conejo se para sobre la malla), el sistema de disparo se libera de forma manual jalando un hilo de monofilamento que va hasta el sitio donde se 
está monitoreando la red. Este sitio generalmente está a unos $25 \mathrm{~m}$ de distancia y puede ser una casa de campaña o un edificio. Una vez que la trampa de red se ha activado, la malla de plástico adopta la forma de bolsa, donde se mantiene al conejo capturado (Figura 2).

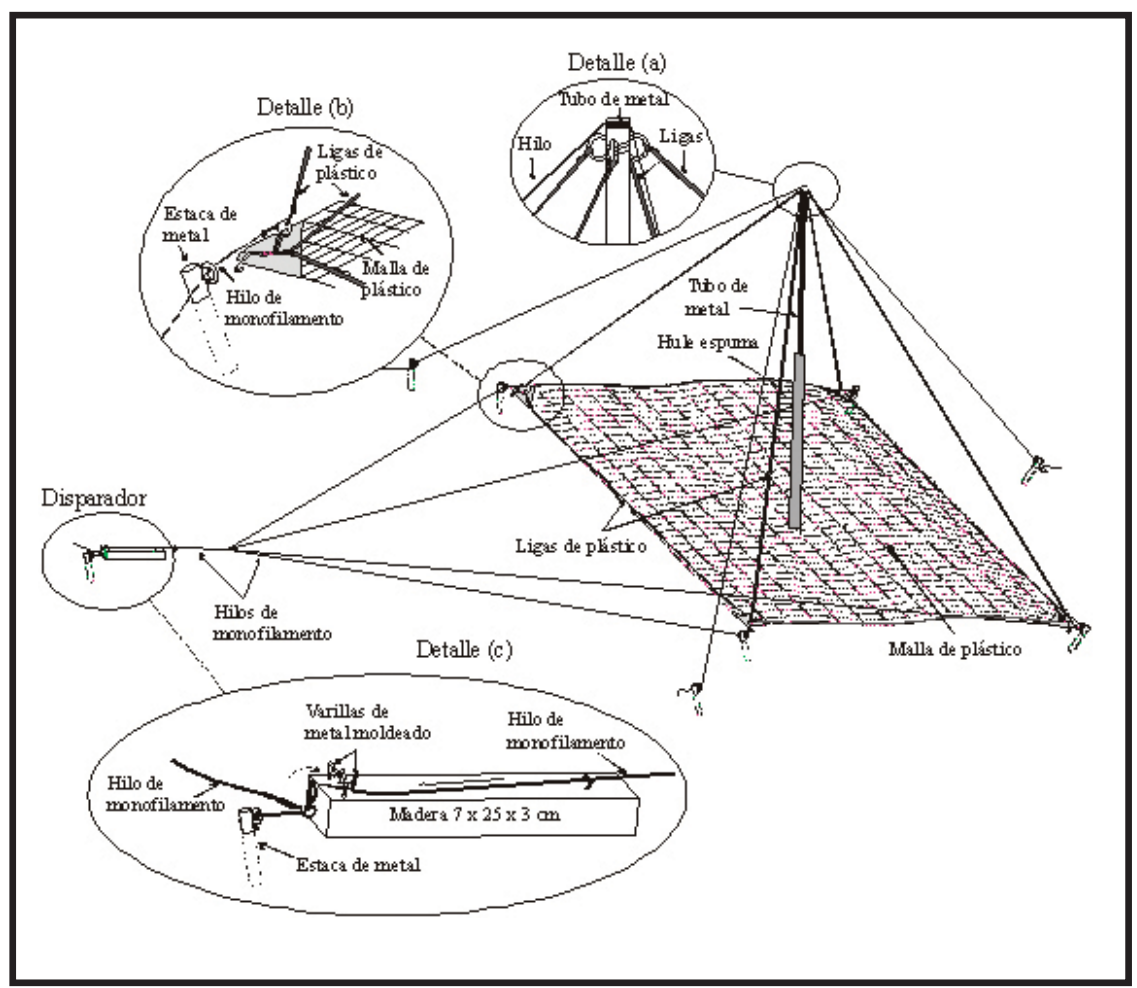

Figura 1. Esquema del diseño de la trampa de red donde se señalan las diferentes partes y detalles que la integran. 


\section{PRUEBAS}

De mayo de 2000 a agosto de 2002 se emplearon 143 noches ( 5 noches en promedio por mes) de muestreo. En la captura de conejos vivos de ambas especies se emplearon trampas de caja metálica (Tomahawk® Live Trap Co. WI, USA, \# 106 y 108) y las trampas de red. En cada uno de los tres sitio se colocaron de 16 a 31 trampas de caja y de 3 a 6 trampas de red empleando alfalfa fresca como cebo (Medicago sativa), se procuró que ambos tipos de trampas quedaran separadas una de la otra para evitar interrupciones con las revisiones y las observaciones, respectivamente. Los dos tipos de trampas se movieron de lugar entre los tres sitios de captura a intervalos de dos o tres noches. Las trampas de caja fueron colocadas entre los pastos y a un lado de los caminos de cada sitio. Las trampas de red fueron colocadas en claros naturales ya que se requería de mayor espacio de superficie en cada sitio. Se emplearon 2536 noches trampa de caja (número de trampas usadas x número de noches) y 644 noches trampa de red durante todo el estudio.

Las trampas tanto de caja como de red fueron activadas y monitoreadas entre las 16:00 h y las 9:00 h del día siguiente. Las trampas de caja fueron revisadas cada dos horas haciendo un recorrido con una lámpara de mano y una bolsa de tela para meter a los conejos, en caso de capturar alguno. Este intervalo de cada dos horas entre una

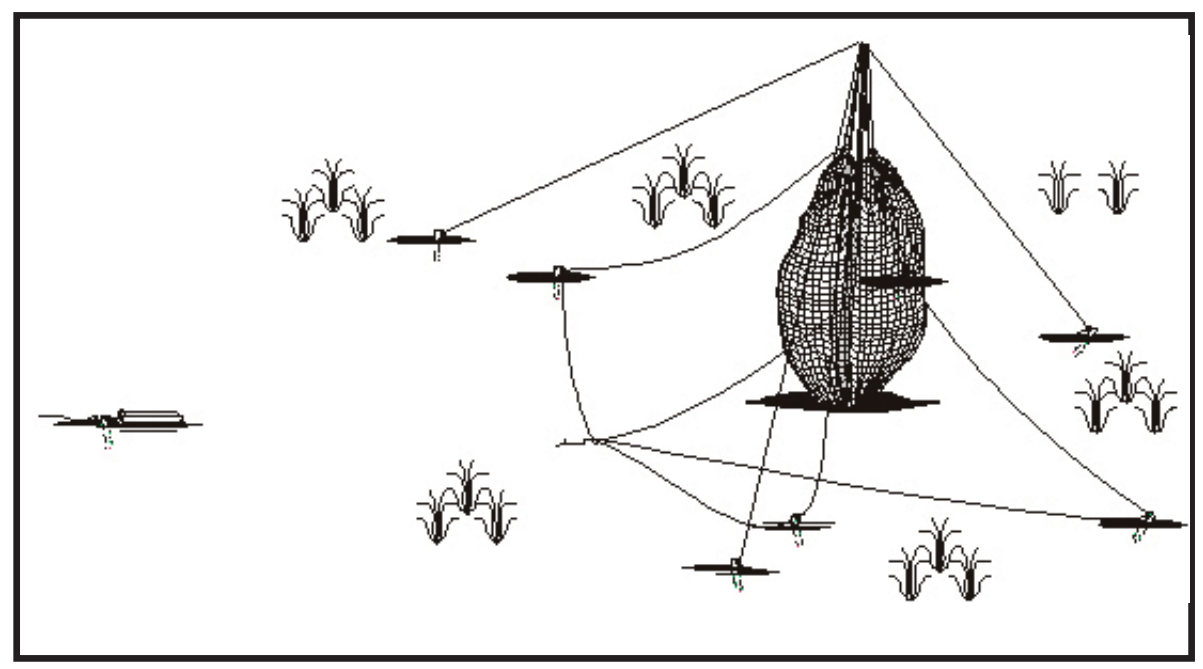

Figura 2. Esquema de la trampa de red después de ser accionada. 
revisión y otra se estableció tratando de disminuir el tiempo de permanencia de los conejos en las trampas y con ello reduciendo las lesiones. Las trampas de red se observaban cada hora, desde una casa de campaña o un edificio, empleando binoculares ópticos (Pentax ${ }^{\circledR}$ 10x50) durante el día, o de visión nocturna (Night Owl Vision ${ }^{\circledR} 4 \mathrm{x}$ ) durante la noche. Algunas ocasiones se emplearon binoculares ópticos para revisar trampas de red durante la noche, amarrando en el tubo de metal central de la red un foco de 12 volts energizado con un acumulador de automóvil. Cuando algún conejo subía a una de las redes, el hilo de monofilamento que va de la red al disparador se jalaba vigorosamente desde el lugar de observación, entonces la red se accionaba dejándola en forma de bolsa impidiendo así que el conejo escapara (Figura 2; Figura 3).
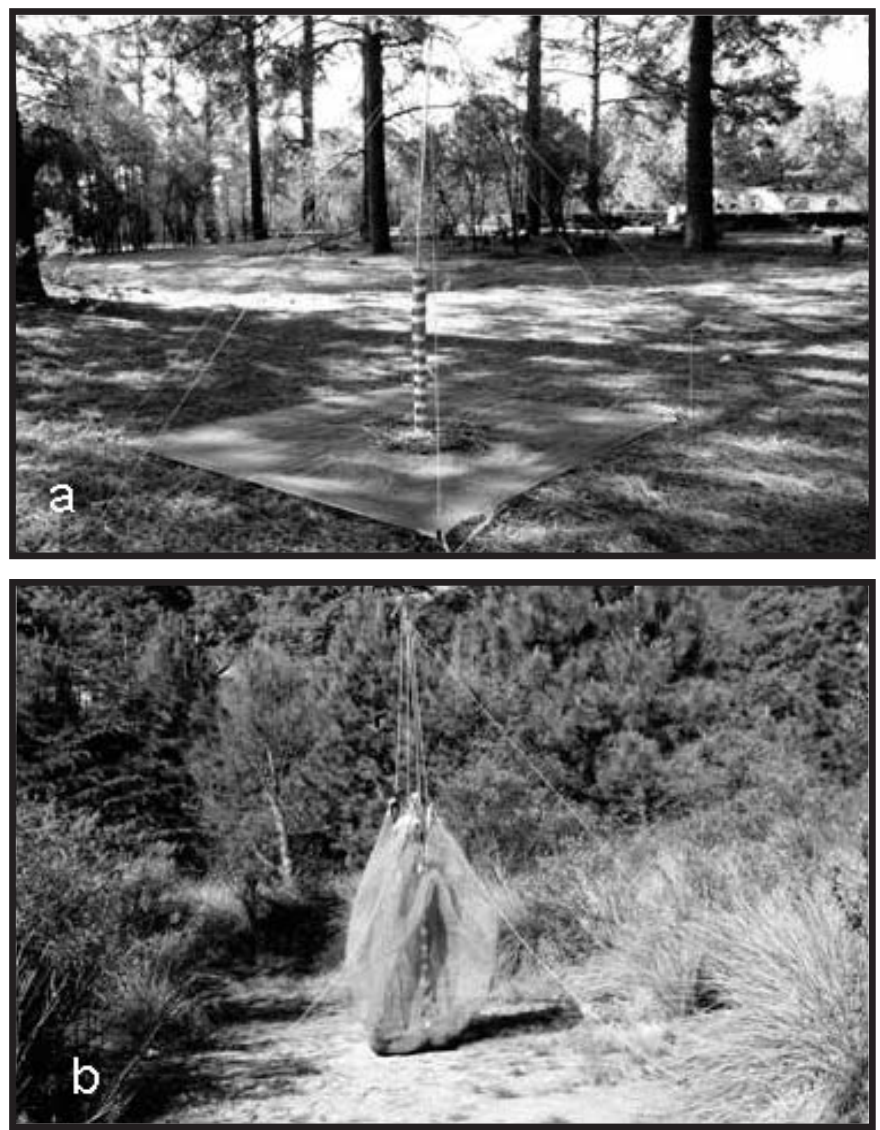

Figura 3. a) Red lista para ser activada. b) Red después de haber sido activada. 
Una vez capturado un conejo en la trampa de red o detectado en la trampa de caja fue inmediatamente introducido a una bolsa de tela para trasladarlo a un área segura donde se les marcó para su identificación en las orejas con un arete y un tatuaje, antes de ser liberado en el mismo sitio donde fue capturado. Se registró el día de captura, especie, sexo y peso corporal usando una balanza de 5 g de precisión.

De acuerdo con los datos obtenidos se evaluaron algunos parámetros que permitieron comparar el funcionamiento entre los dos tipos de trampas según Luengos Vidal et al. (2003). Los parámetros fueron: 1) eficiencia de captura (número de individuos atrapados / número de noches trampa), 2) eficiencia de recaptura (frecuencia de individuos capturados más de una vez, clasificados como sólo capturados en trampas caja, sólo en red e individuos recapturados tanto en caja como en red y, 3) sesgo de captura respecto al sexo y peso corporal. En trabajos previos hemos establecido una correlación entre peso corporal y estado reproductor lo cual nos ha dado un aproximado de la edad (González et al., 2007; Vázquez et al., 2007) dado que los métodos para estimar apropiadamente la edad en individuos vivos en el género Sylvilagus no están establecidos. En el presente trabajo dividimos a los animales capturados en tres categorías de peso elegidas de la misma forma arbitraria. Se calcularon tres rangos con los valores de peso corporal de los individuos capturados en su primera ocasión así que en S. cunicularius la categoría "uno" correspondió a individuos con un peso menor a los $1135 \mathrm{~g}$, la "dos" a individuos de $1136 \mathrm{~g}$ hasta 1745 g y la "tres" a individuos con un peso mayor a 1745 g. En S. floridanus la categoría "uno" correspondió a individuos con un peso menor a los 811.7 g, la "dos" a individuos de $811.8 \mathrm{~g}$ hasta $968.4 \mathrm{~g}$ y la "tres" a individuos con un peso mayor a 968.4 g. El porcentaje de conejos capturados en cada categoría de peso fue comparados entre cada una de las tres categorías de peso empleando pruebas de $\mathrm{X}^{2}$, para los conejos capturados en cada tipo de trampa respectivamente.

Los tipos de lesiones que presentaron los conejos al momento de ser capturados tanto en trampas de caja como de red fueron descritos de forma cualitativa. Los análisis estadísticos incluyeron estadística descriptiva y pruebas de U de MannWhitney para comparar la eficiencia de captura y Pruebas de $\mathrm{X}^{2}$ (cuando las proporciones fueron menores al $5 \%$, los datos se analizaron empleando una pruebas de probabilidad exacta de Fisher de dos colas) para comparar el porcentaje de recapturas y de categorías de peso. Todos los análisis se hicieron con el paquete estadístico GraphPad InStat versión 3.00 para Windows, Software Inc. San Diego California USA, www.graphpad.com, en el Centro Tlaxcala de Biología de la Conducta UAT-UNAM.

\section{RESULTADOS}

Durante el período de mayo del 2000 a agosto del 2002, se realizaron 164 capturas de 90 conejos de $S$. cunicularius ( 47 hembras y 43 machos) y 28 capturas de 16 S. floridanus 
(6 hembras y 10 machos). Casi todos los conejos capturados se pusieron en libertad, excepto por cuatro hembras y dos machos de $S$. cunicularius que fueron colectados.

\section{Eficiencia}

En S. cunicularius la eficiencia para capturar por primera vez a los conejos (incluyendo hembras y machos) empleando trampas de caja tuvo una media de 0.004 (mínimo $=0.0$, máximo $=0.035$ ) y al emplear trampas de red de 0.091 (mínimo $=0.045$, máximo $=0.155$ ) La eficiencia de captura entre ambos métodos presentó diferencias estadísticamente significativas $(U=705.5, p>0.0001)$, siendo las trampas de red 22 veces más eficientes que las de caja.

En S. floridanus la eficiencia de captura al emplear trampas de caja tuvo una media de 0.006 (mínimo $=0.0$, máximo 0.028) y al emplear trampas de red de 0.008 ( mínimo $=0$, máximo $=0.061$ ). Las diferencias en la eficiencia de captura entre ambos tipos de trampa no fueron estadísticamente significativas $(U=70.5, p=0.95)$.

Se capturaron en más de una ocasión a 35 conejos de $S$. cunicularius (19 hembras y 16 machos), de los cuales el $3 \%$ (un individuo) fue recapturado sólo en trampas de caja, el 66 \% (23 individuos) sólo en trampas de red y el 31 \% (11 individuos) en ambos tipos de trampa. Las diferencias en la proporción de individuos recapturados sólo en trampas de caja y los recapturados sólo en las de red fueron estadísticamente significativas (Fisher $=1.91, p<0.0001$ ). De los conejos recapturados tanto en trampas de caja como en las de red se obtuvo la frecuencia de recaptura para cada tipo de trampa. La frecuencia con la que se recapturaron conejos en red (media $=$ 2, mínimo = 1, máximo = 7) fue mayor a la obtenida en trampas de caja (media = 1, mínimo $=1$, máximo $=3$ ), siendo las diferencias estadísticamente significativas $($ Wilcoxon = $-21, p=0.031)$.

Se capturaron en más de una ocasión a seis conejos de $S$. floridanus (2 hembras y 4 machos), de los cuales un individuo fue capturado sólo en trampas de caja, cuatro en trampas de red (un individuo con tres recapturas y tres con sólo una) y uno en ambos tipos de trampa (tres recapturas en trampas de caja y tres en red). Debido al reducido tamaño de muestra los datos no fueron analizados estadísticamente.

\section{Sesgo de captura respecto al sexo y al peso}

En S. cunicularius la proporción de sexos obtenida de individuos capturados por primera vez al emplear trampas de caja no fue distinta de $1: 1\left(x^{2}=1.63, p=0.2\right)$. La misma proporción de sexos se encontró al emplear trampas de red $\left(x^{2}=0.02, p=0.88\right)$.

En S. floridanus, la proporción de sexos al emplear trampas de caja fue sesgada hacia los machos (proporción 7:3), siendo las diferencias estadísticamente significativas $\left(x^{2}=8.33, p=0.003\right)$. En discrepancia con lo anterior, la proporción de 
sexos obtenida al emplear trampas de red no mostró diferencias estadísticamente significativas de la proporción 1:1 $\left(x^{2}=0.72, p=0\right.$. 39).

En S. cunicularius las proporciones de individuos capturados en cada una de las categorías de peso al emplear trampas de caja fueron del $30 \%$ (ocho individuos) para la categoría "uno", el 48 \% (13 individuos) para la categoría "dos" y el 22 \% (seis individuos) para la categoría "tres". Las diferencias en las proporciones no fueron estadísticamente significativas $\left(x^{2}=5.11, p=0.08\right)$. Al emplear trampas de red las proporciones obtenidas para cada categoría correspondieron al $5 \%$ (tres individuos) para la categoría "uno", 47 \% (29 individuos) para la categoría "dos" y 48 \% (30 individuos) para la categoría "tres". Las diferencias en dichas proporciones fueron estadísticamente significativas ( $x^{2}=28.86, p<0.0001$ ), siendo en la categoría "uno" donde una menor proporción de individuos fueron capturados.

En S. floridanus la proporción de conejos capturados para cada una de las categorías de peso al emplear trampas de caja correspondió al 43 \% (tres individuos) para la categoría "uno", 57 \% (cuatro individuos) para la categoría "dos" y para la categoría "tres" no se logró capturar ningún individuo. Las diferencias de las proporciones entre las tres categorías de peso fueron estadísticamente significativas $\left(x^{2}=40.71, p<0.0001\right)$. Al emplear trampas de red las proporciones obtenidas para cada categoría de peso correspondieron al $45 \%$ (cuatro individuos) para la categoría "uno", 33 \% (tres individuos) para la "dos" y 22 \% (dos individuos) para la "tres". Estas diferencias no fueron estadísticamente significativas $\left(x^{2}=4.04, p=0.13\right)$.

\section{Lesiones causadas al momento de la captura}

Las lesiones más comunes de los conejos de S. cunicularius y S. floridanus capturados en trampas de caja incluyeron el desprendimiento de pelo en la frente, en las patas y en los brazos a causa de los repetidos golpes que ellos se dan contra las paredes de la trampa. En casos más extremos los golpes causaron que la piel se abriera en regiones como las patas traseras y principalmente los labios y la frente. Las lesiones más graves se encontraron en la frente, habiendo dos casos donde algo de piel se desprendió de los huesos frontales.

Las lesiones más comunes para ambas especies de conejos al emplear trampas de red incluyeron el desprendimiento de pelo en la parte dorsal cerca de la cola, en las patas traseras y en las orejas a causa de caer al suelo después de saltar contra la red. La lesión más grave fue una de labios rotos con algo de sangre.

\section{DISCUSIÓN}

Las capturas con los dos tipos de trampas presentaron una especificidad del $100 \%$ exclusivamente para las dos especies de conejos (S. cunicularius y S. floridanus) 
reportadas en el Parque Nacional La Malinche (Martínez-Chacón et al., 2005; Fernández, 2005; González et al., 2007; Vázquez et al., 2007). Esta especificidad puede estar dada por el cebo empleado (alfalfa), el cual fue atractivo sólo para los conejos. La especificidad de las trampas de red para capturar lagomorfos fue corroborada durante un estudio del parasitismo en liebres (Lepus californicus) y conejos del desierto ( $S$. audubonii), en la reserva de la biosfera de Mapimí, Durango, donde ambas especies fueron capturadas eficientemente (Amorós et al., 2006; J. Vázquez, com. pers).

En este estudio, a pesar de utilizar un mayor esfuerzo de captura con las trampas de caja, las trampas de red fueron más eficientes para capturar y recapturar conejos de $S$. cunicularius. Varias razones no excluyentes pudieran explicar dichas diferencias. En primer lugar, las trampas de red al ser colocadas sobre el suelo en lugares donde la vegetación arbustiva es escasa, probablemente permitió a los conejos observar más fácilmente el cebo y ser atraídos de forma más efectiva. En contraste, las trampas de caja fueron colocadas principalmente entre los pastizales o sólo cerca de áreas con escasa vegetación arbustiva por lo que quizá el cebo fuera menos visible para conejos que realizan sus actividades en zonas con menor vegetación lo que resultó en una menor eficiencia de captura. Otra posibilidad es que los conejos para obtener alimento tengan más aversión a entrar en objetos extraños, como las trampas de caja, por lo que las superficies planas con cebo, como las que ofrecen las trampas abiertas de red, puedan representar un menor riesgo para los conejos. Esta posibilidad es apoyada por una mayor frecuencia de recapturas en trampas de red que en trampas de caja, lo cual sugiere que los conejos se arriesgaron más a acercarse a este tipo de trampas. Existe la posibilidad de que la menor eficiencia de captura en las trampas de caja sea debida a las revisiones hechas cada dos horas. Sin embargo, durante las revisiones de las trampas se observaba frecuentemente una disminución del cebo colocado previamente como consecuencia de que los conejos lo estuvieron consumiendo. Ello sugiere que dichas revisiones no afectaron mucho el acercamiento de los conejos a las trampas. Además, hubo ocasiones que tan solo unos minutos después de suministrar más cebo a las trampas, se obtuvieron capturas. El sesgo de capturas en las trampas de red hacia las dos categorías de individuos más pesados, sugiere que los individuos adultos probablemente sean los más arriesgados a acercarse a las trampas de red. Además, las trampas de red fueron colocadas sólo en claros naturales, donde hay más actividad de los individuos adultos (J. Vázquez, com. pers.), tal como se reporta para S. floridanus (Lorenzo y Cervantes, 2005).

En S. floridanus no se obtuvieron diferencias en la eficiencia de captura entre ambos tipos de trampas y no se logró evidenciar algún patrón en la eficiencia de recaptura posiblemente debido al reducido tamaño de muestra. Asimismo, los resultados obtenidos de la proporción de capturas respecto al peso muestran que la categoría de mayor peso no fue representada. Sin embargo no fue posible capturar un mayor número de individuos para analizar estadísticamente los datos. 


\section{Sesgo de captura respecto al sexo de los individuos}

En S. cunicularius, la proporción de sexos obtenida de los conejos capturados con trampas de caja y red en este estudio fue 1:1. La misma proporción de sexos en esta especie fue reportada tres años después, en un estudio donde sólo se emplearon trampas de red (González et al., 2007). En S. floridanus, al emplear trampas de red la proporción de sexos fue 1:1, similar a la proporción 1:1 reportada para conejos de $S$. floridanus capturados con trampas de caja en Oregon, Estados Unidos (Chapman y Trethewey, 1972). Mientras tanto, al emplear trampas de caja se capturó una mayor proporción de machos que de hembras (7:3). Algo similar se ha encontrado en el conejo europeo Oryctolagus cuniculus, donde la proporción de machos es mayor al de las hembras cuando se emplean trampas de caja (Smith et al., 1995). Estos resultados señalan los beneficios de cada tipo de trampa y la necesidad de emplear distintos tipos de ellas para obtener una mejor representación de la dinámica poblacional de los modelos de estudio en cada investigación.

Las ventajas de emplear las trampas de red para capturar conejos silvestres incluyen obtener mayor eficiencia de captura y recaptura, una proporción no desviada hacia un sexo, mayor proporción de individuos adultos y, principalmente, que causa menos lesiones a los animales. Por lo contrario, emplear las trampas de red implica que los sitios para colocarlas están restringidos a áreas despejadas, o bien invertir tiempo para abrir tales áreas. Asimismo, el tiempo aproximado de colocar una trampa de red es de aproximadamente 15 minutos y se requiere mayor esfuerzo humano para monitorear este tipo de trampas ya que, en nuestro caso, una persona sólo puede monitorear máximo hasta cinco trampas simultáneamente. Sin embargo, este mayor esfuerzo se compensa con la mayor eficiencia de la trampa red, ya que puede resultar mayor esfuerzo estar revisando las trampas de caja cada dos horas.

\section{CONCLUSIÓN}

Los resultados de evaluar el desempeño de las trampas de red para capturar conejos silvestres en el Parque Nacional La Malinche, Tlaxcala, muestran que son eficientes para capturar lagomorfos vivos, sin provocar severos daños físicos. Por lo tanto, el desarrollo de nuevos diseños de captura, como las trampas de red, es una alternativa que puede tener mayores beneficios para estudios enfocados al manejo y conservación de fauna que otros métodos tradicionales.

\section{AGRADECIMIENTOS}

A Humberto Pérez, Bernardo Romero, Minerva Flores y Fernando Aguilar por su colaboración en el trabajo de campo. A Laura García por su excelente apoyo técnico en 
las instalaciones de la ECLM y a Carolina Rojas en la búsqueda bibliográfica y gestión administrativa. Financiamiento IIB-UNAM, PROMEP UATLX-CA-191.

\section{LITERATURACITADA}

Amorós, D.M., D. Romero, J.A. Guerrero y A.J. Martínez-Chacón. 2006. Prevalencia de ectoparásitos en los lagomorfos de la reserva de la biosfera de Mapimí, Durango. VIII Congreso Nacional de Mastozoología. Zacatecas, Zac. Noviembre 27 - Diciembre 01. C10.

Ceballos, G. 2005. Orden Lagomorpha. Pp. 825-850, en: Los Mamíferos Silvestres de México (G. Ceballos y G. Oliva, eds.). Comisión Nacional para el Conocimiento y Uso de la Biodiversidad y Fondo de Cultura Económica. México, D.F.

Cervantes, F.A. 1993. Conejos y Liebres Silvestres de México. Ciencia y Desarrollo, 10:58-69.

Chapman, J.A. y D.E.C. Trethewey. 1972. Factors affecting trap responses of introduced eastern cottontail rabbits. Journal of Wildlife Management, 36:1221-1226.

Craven, S.R. 1994. Cottontail rabbits. Pp. 75-80, en: Prevention and Control of Wildlife Damage (S.E. Hygnstrom, R.M. Timm y G.E. Larson, eds.) Vol. 2. University of Nebraska, Lincoln, Nebraska, USA.

Fernández, J.A. 2005. Mamíferos. Pp. 137-56, en: Biodiversidad del Parque Nacional Malinche. Tlaxcala, México (J.A. Fernández y J.C. López, eds.). Coordinación General de Ecología del Estado de Tlaxcala. México.

González, J.,C. Lara, J. Vázquez y M. Martínez-Gómez. 2007. Demography, density, and survival of an endemic and near threatened cottontail Sylvilagus cunicularius in central Mexico. Acta Theriologica, 52:299-305.

Lorenzo, C. y F.A. Cervantes. 2005. Sylvilagus floridanus (J.A. Allen, 1890). Pp.843-845, en: Los Mamíferos Silvestres de México (G. Ceballos y G. Oliva, eds.). Comisión Nacional para el Conocimiento y Uso de la Biodiversidad y Fondo de Cultura Mexicana. México, D.F.

Luengos Vidal, E.M., M. Lucherini y E. Casanave. 2003. An evaluation of tree restraining devices for capturing pampas foxes. IUCN/SSC Canid Specialist Group. Canid News, 6:1

URL: http://www.canids.org/canidnews/6/Devices_for_capturing_pamapas_foxes.pdf

Martínez-Chacón, A.J., E. Rivera, J. Vázquez, L. Rodríguez-Martínez y M. Martínez-Gómez. 2005. El papel ecológico de los lagomorfos: interacciones interespecíficas, biología reproductora y comportamiento. Pp. 199-210, en: Biodiversidad del Parque Nacional Malinche. Tlaxcala, México (J.A. Fernández y J.C. López, eds). Coordinación General de Ecología del Estado de Tlaxcala. México.

Powell, R.A. y G. Proulx. 2003. Trapping and marking terrestrial mammals for research: Integrating ethics, performance criteria, techniques, and common sense. Institute for Laboratory Animal Research Journal, 44:259-276.

Smith G.C., B. Pugh y R.C. Trout. 1995. Age and sex in samples of wild rabbits, Oryctolagus cuniculus, from wild populations in southern England. New Zealand Journal of Zoology, 22:115-121. 
Thurston, S.N. y M.C. Brittingham. 1997. Wildlife Damage Control \# 7: Cottontail rabbits. Penn States of Agricultural Sciences. The Pennsylvania State University. URL: http:// pubs.cas.psu.edu/freepubs/pdfs/uh095.pdf

Vanzolini, P.E. y N. Papayero. 1990. Manual de recolección y preparación de animales. México, D.F. Universidad Nacional Autónoma de México.

Vázquez, J., A.J. Martínez Chacón, R. Hudson, L. Rodríguez-Martínez y M. Martínez Gómez. 2007. Seasonal reproduction in Mexican cottontail rabbits Sylvilagus cunicularius in La Malinche National Park, central Mexico. Acta Theriologica, 52:361-369. 